\title{
Study of Optical Output Couplers for Submillimeter Wavelength Backward-Wave Oscillators (BWO's)
}

Jerry D. Cook

Eastern Kentucky University

Richmond, Kentucky

Norbert Stankiewicz

National Aeronautics and Space Administration

Lewis Research Center

Cleveland, Ohio

and

Mark Podany

Analex Corporation

Lewis Research Center

Cleveland, Ohio

Prepared for the

13th International Conference on Infrared and Millimeter Waves sponsored by Society of Photo-Optical and Instrumentation Engineers Honolulu, Hawaii, December 5-9, 1988

\section{Nush}

(AASA-TH-1C1:60) SIUCY CE CETICAL CUIEUI

N89-111<8 



\author{
STUOY OF OPTICAL OUTPUT COUPLERS FOR SUBMILLIMETER \\ WAVELENGTH BACKWARD-WAVE OSCILLATORS (BWO'S) \\ Jerry D. Cook \\ Department of Physics \\ Eastern Kentucky University \\ Richmond, Kentucky 40475 \\ Norbert Stankiewicz \\ National Aeronautics and Space Administration \\ Lewis Research Center \\ Cleveland, Ohio 44135 \\ and \\ Mark Podany \\ Analex Corporation \\ Lewis Research Center \\ Cleveland, Ohio 44135
}

\title{
Abstract
}

The machining of slow wave structures for high frequency BWO's is extremely difficult beyond 1 THz. Recently a microfabrication technique using photolithography and ion-beam assisted etching has been used to construct a prototype BWO operating at 200 to $265 \mathrm{GHz}$.

The output coupler for such tubes remains a problem. Waveguides do not exist or are very lossy at the frequencies of interest ( 300 to $2000 \mathrm{GHz}$ ).

This paper discusses several scaled experiments of optical output couplers for submillimeter BWO's. Various designs of planar antennas (Vivaldi horns) and lens-feed systems (hyperhemispherical lens) were constructed and tested between 20 and $100 \mathrm{GHz}$ using a spectrum analyzer. The lens system was also tested at $337 \mathrm{GHz}$ using a $\mathrm{CO}_{2}$ pumped FIR laser.

Optical coupling; Submillimeter BWO's; Planar antennas Electron beam tubes; Local oscillators 


\section{Introduction}

NASA Lewis Research Center is directing a program to develop a series of backward-wave oscillators for the frequency range 300 to $2000 \mathrm{GHz}$. The intention is to use the BWO's as local oscillators for heterodyne receiver spectrometers in a space-based astronomical observatory.

Because the characteristic dimensions of slow wave structures for electron beam tubes are of the order of fractions of wavelengths, traditional fabrication methods become difficult at submillimeter wavelengths. Although machined slow wave structures have been reported for BWO's operating at $1 \mathrm{THz}$ (ref. 1) and $1.5 \mathrm{THz}$ (ref. 2) these were limited to the relatively easy to construct vanes known as Millman structures. However, Millman structures are forward-wave fundamental structures that operate as oscillators only in the backward-wave portion of their dispersion curve where the interaction impedance is substantially reduced thus requiring higher electron beam powers. The interdigital line used in this work is a backward-wave fundamental structure that has a higher interaction impedance therefore requiring less electron beam power to initiate oscillation. This structure also has the advantage of having a larger tunable bandwidth.

The photolithographic technique in reference 3 was used to microfabricate a folded slot line for a BWO designed to operate between 200 and $265 \mathrm{GHz}$. The BWO was successfully tested as reported in reference 4 . A WR-3 waveguide was used as the output port in this experiment. Figure I shows the design of the etched interdigital line and the coplanar waveguide (used as a transition between the slot line of the circuit and the WR-3 waveguide). However, the coupling losses from the folded slot line to the outside window was estimated to be $10 \mathrm{~dB}$. At the higher frequencies, where lower output powers are expected, the inefficiency of such a system cannot be tolerated.

This study was initiated to experimentally evaluate various slot line/planar horn designs that could be incorporated onto the substrate with the slow-wave structure. The radiation launched by the planar antenna will feed a 


\section{ORIGNAL PAGE IS \\ OF POOR QUALITY}

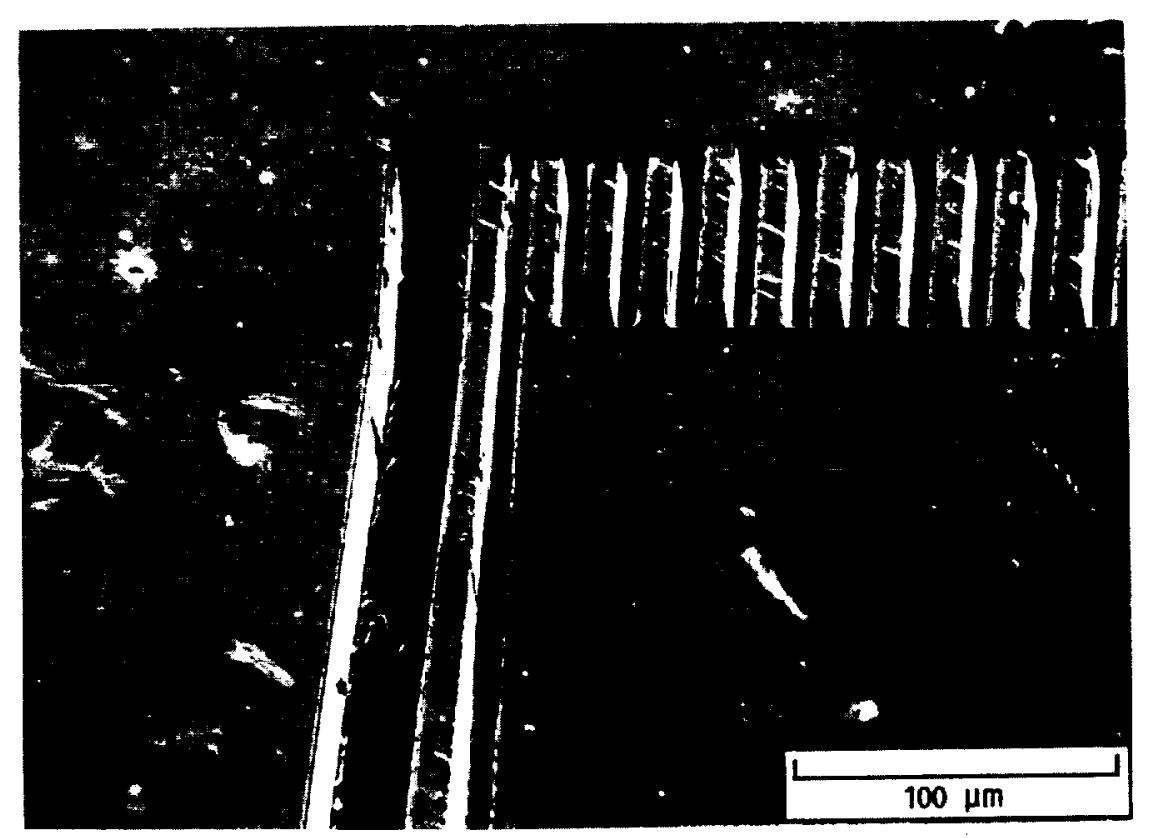

FIG. 1 SEM PHOTOGRAPH OF ETCHED SLOW-WAVE STRUCTURE SHOWING INTERDIGITAL LINE AND CO-PLANER MAVEGUIDE OUTPUT COUPLER.

hyperhemispherical lens and a subsequent collimating lens will direct the energy through the vacuum window of the BWO. The lens system is shown in figure 2 .

Most of the literature on planar antennas deals with spatial patterns at a single frequency, in this paper we concentrate primarily on the frequency response of the various designs. Because of the large tunable bandwidths of the BWO's we are interested in developing an output coupler which has a flat frequency response across the band.

\section{Experimental Setup for Scaled Tests}

The scaled experiments were carried out between 30 and $40 \mathrm{GHz}$ and between 85 and $100 \mathrm{GHz}$. Figure 3 shows the experimental setup.

All the experiments were normalized to the double horn reference experiment shown in figure $4(a)$. The frequency 


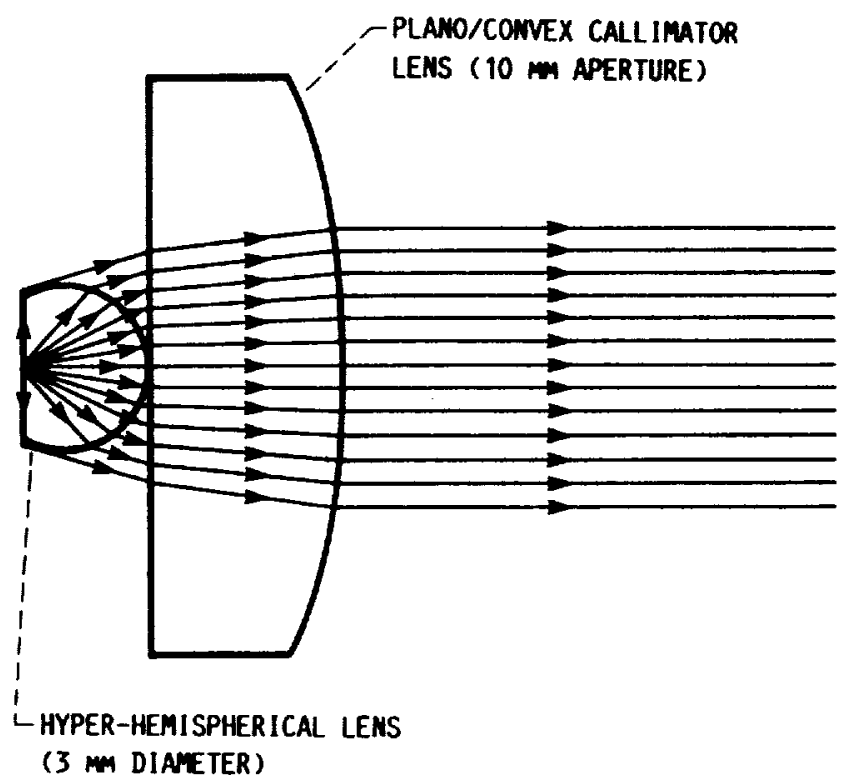

FIG. 2 RAY TRACE OF HYPER-HEMISPHERICAL LENS AMD COLLIMATING LENS.

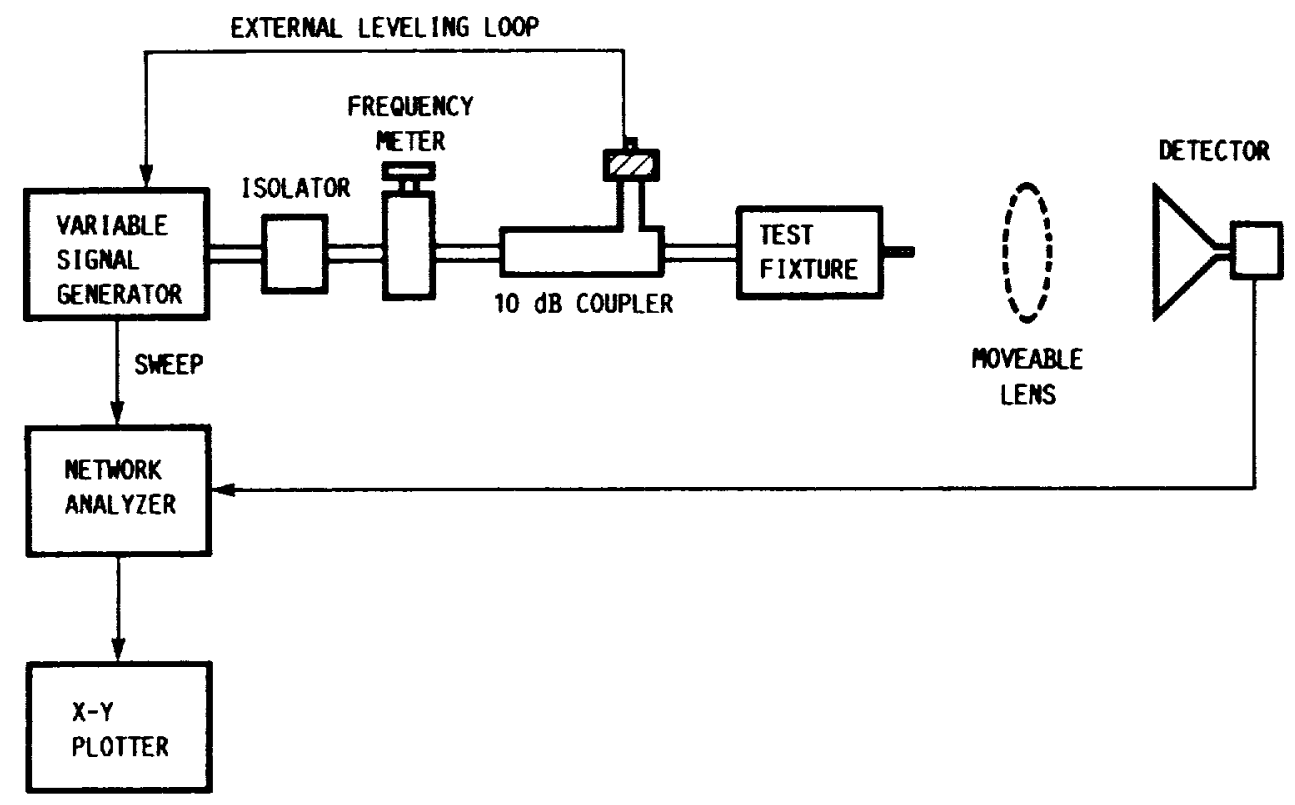

FIG. 3 DIAGRAM OF EXPERIMENTAL ARRANGEMENT FOR SCALED TESTS AT 30-40 GHZ AND AT 85-100 GHz. 


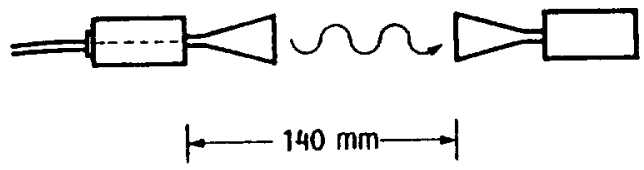

(a) DOUBLE HORN SETUP WITH SLOT LIME LOCATED IN WAVEGUIDE.

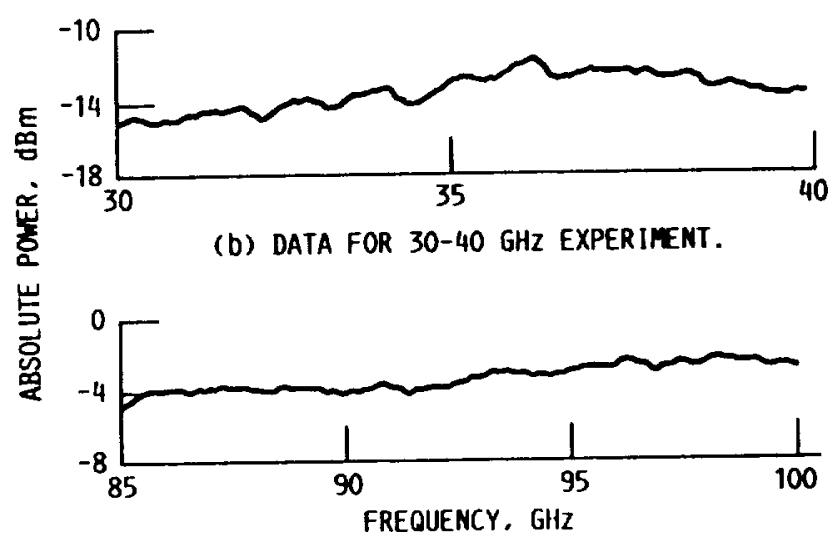

(c) DATA FOR 90-100 GHz.

FIG. 4 REFERENCE EXPERIMENTS.

response of the reference experiments at 30 to $40 \mathrm{GHz}$, figure $4(b)$, and at 85 to $100 \mathrm{GHz}$, figure $4(c)$, were periodically remeasured and stored in the spectrum analyzer.

Figure 5 is a sketch of the test fixtures showing typical horn, slot line, and input waveguide transition to the slot line. The test sections were wet etched on copper clad Duroid ( $5 \mathrm{mils}$ thick) and mounted into a WR-28 waveguide for the 30 to $40 \mathrm{GHz}$ experiments; and into a WR-10 waveguide for the 85 to $100 \mathrm{GHz}$ experiments.

The Vivaldi planar antenna, see e.g., reference 5 , opens exponentially as,

$$
W=g \exp \{k x\}
$$

where $g$ is the gap width of the slot line and $k$ is determined from

$$
k=\frac{1}{L} \ln \frac{W_{0}}{g}
$$




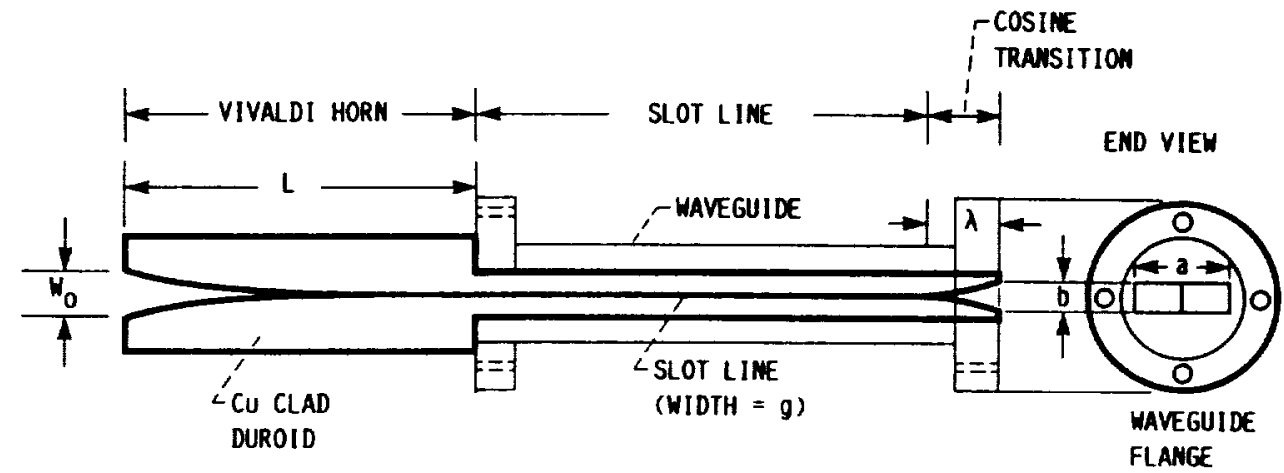

FIG. 5 SKETCH OF WAVEGUIDE/SLOT LINE TEST FIXTURE WITH COSINE TRANSITION AND VIVALDI HORN ANTENMA.

In equation (2) $L$ is the length of the horn and $W_{0}$ is the final opening. Horns were constructed and studied with lengths $L=3 \lambda, 4 \lambda$ and $w_{0}=\lambda / 2, \lambda, 2 \lambda, 3 \lambda$ (where $\lambda$ was chosen as the wavelength at $30 \mathrm{GHz}$ and at $90 \mathrm{GHz}$ ).

Usually Vivaldi horns have lengths of about 3 to $10 \lambda$ (see e.g., ref. 6). The space available on the substrate containing the slow wave structure limited us to the study of shorter horns.

A cosine transition is used between the waveguide and the slot line:

$$
y=\left[\frac{b-q}{2}\right] \cos \left(\frac{\pi x}{2 \lambda}\right)
$$

where $b$ is the waveguide dimension; see figure 5 . These finline transitions are on the order of a wavelength to provide a smooth transition between the slot line and the waveguide (ref. 7).

The gap width of the slot line in the scaled experiments was determined from the analysis given in reference 8 . The Duroid substrate is $5 \mathrm{mil}$ thick and the slot width was $4 \mathrm{mil}$ for all the scaled tests. This is consistent with the field requirements in the above reference but it is not a properly scaled width for the interdigital structure used in the BWO. However, because all the measured data presented here is normalized to a reference experiment having the same slot width, the frequency characteristics of the slot itself would be divided out by the normalization. 


\section{Scaled Test Results}

Tests at 30 to $40 \mathrm{GHZ}$

Reference experiment. - All results obtained at 30 to $40 \mathrm{GHz}$ were compared to the reference displayed in figure $4(b)$. This allows us to make comparative measurements and evaluate individual microstrip antennae against a common standard.

The effect of mouth width. - Figure 6 illustrates the effects of changing $W_{0}$, the maximum opening of the horn. In this figure the length of the horn was held constant at $40 \mathrm{~mm}$ (about $4 \lambda$ at $30 \mathrm{GHz}$ ) and the opening was varied from $1 / 2$ to $3 \lambda$. The horns are evaluated on the flatness of response as a function of frequency as well as the average power detected at normal incidence. The horns get progressively better on both counts as the opening becomes smaller. In fact, the resonance absorbtion bands displayed by horns having larger mouths make them unacceptable. We attribute this structure to defects in the horns, caused either in construction or handling. The horns are very fragile and great care was needed to load the horns into the test fixture. Despite the care small creases in the horns sometimes occurred which resulted in resonance absorption bands as illustrated in the figure.

The frequency response of the smaller horns is somewhat better, although for horns having an opening of only $1 / 2 \lambda$ the response still varied over a $10 \mathrm{~dB}$ range. The average relative power measured for the smaller horn also indicates that smaller horn openings are better. The relative power increased by $20 \mathrm{~dB}$ as $W_{0}$ decreased from 3 to $1 / 2 \lambda$.

The effect of dielectric covers. - Both transmitted power and frequency response of a planar horn is improved by covering the horn with a dielectric. In our study Teflon was used. Figure 7 shows the typical power and frequency response of a horn with two thicknesses of Tefion compared to a bare horn. The thickness of the dielectric was 0.317 and $0.714 \mathrm{~mm}$ or approximately 0.5 and 1.0 of the wavelength within Teflon (permittivity $=2.1$ ). Thinner dielectrics were tried but were ineffective with inconclusive results. 

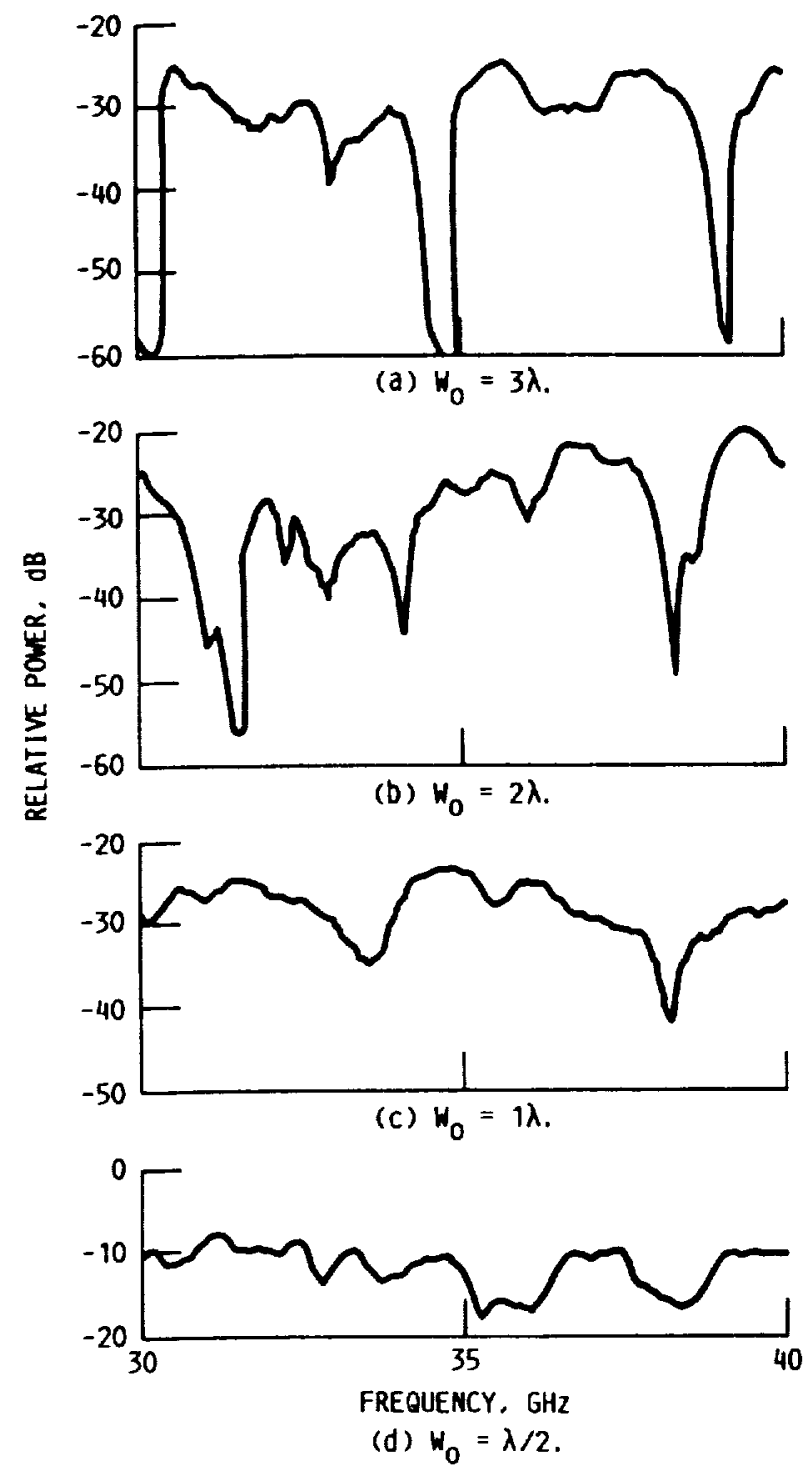

FIG. 6 RELATIVE POHER OUTPUT AS A FUMCTION OF FREQUENCY OF A VIVALDI HORN $(L=4 \lambda)$.

As shown in figure 7 the power level is independent of the thickness of the dielectric, however, the flatness of the frequency response improves considerably with the thicker dielectrics. This was consistent with all horns studled. We were able to improve average power responses by 15 to $20 \mathrm{~dB}$ by covering the horns with a thick dielectric. 

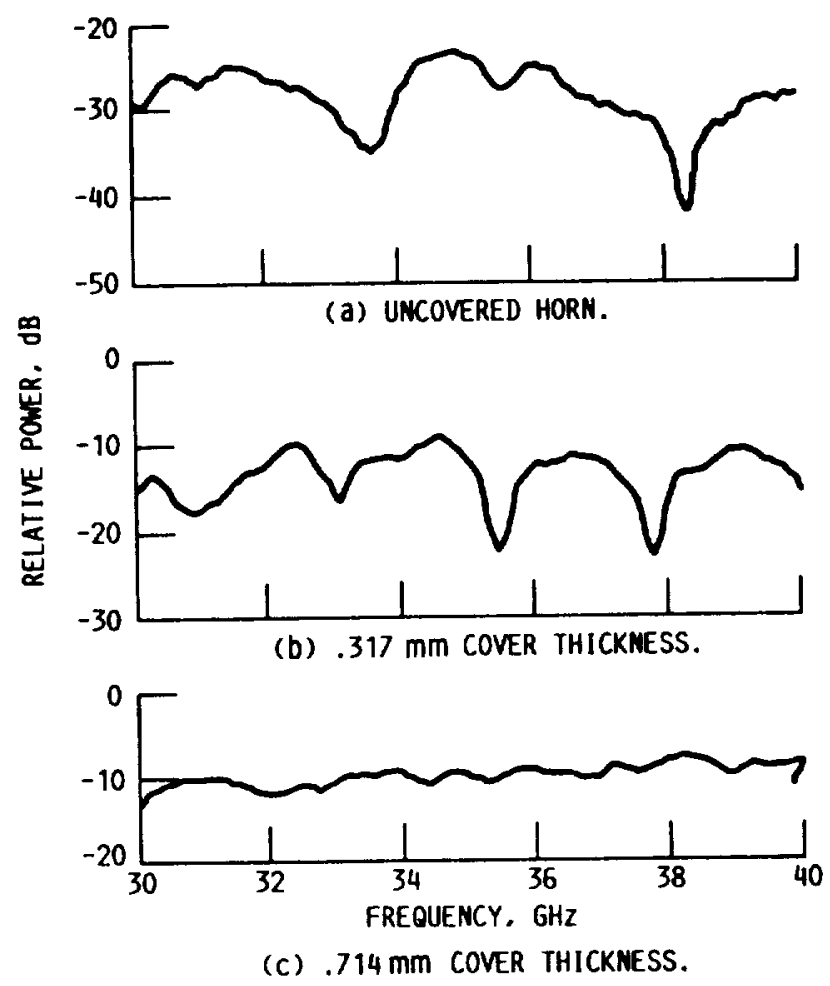

FIG. 7 THE EFFECT OF A DIELECTRIC COVERING (TEFLON) ON A VIVALDI HORN $(L=4 \lambda$. $W_{0}=(\lambda)$.

There was little effect observed in changing the length of the horns from 3 to $4 \lambda$. The longer horns did tend to give a slightly flatter frequency response with marginally better power. Our intended application of these horns precluded a study of longer horns.

Use of lenses. - Figure $8(a)$ shows the data for a planar horn $4 \lambda$ long with an opening $1 / 2 \lambda$ and covered with $a$ $0.714 \mathrm{~mm}$ Teflon dielectric. A double convex lens constructed of high density polyethylene (HOP) and having a focal length of 2 in. and an aperture of 2 in. was then used to focus the power from the horn onto the detector. As expected, a substantial increase in the average power was realized (about 7 to $10 \mathrm{~dB}$ ).

Figure 8 also shows that there are only minor differences between the shapes of the two curves indicating that 


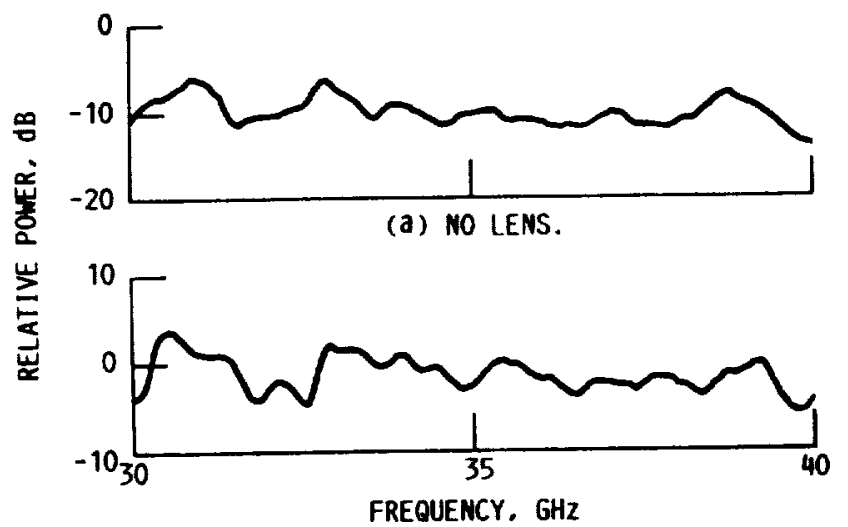

(b) WITH LENS.

FIG. 8 USE OF A LENS IN FOCUSING OUTPUT POWER FROM A VIVALDI HORN $\left(L=4 \lambda, W_{0}=\lambda / 2\right.$, COVER $=.714 \mathrm{~mm}$ THI(K).

a focusing lens can be used to increase the detected power at normal incidence without distorting the frequency response of the horn.

Scaled test of hyperhemispherical lens. - As an alternative to the horns described, a study of a lens coupling system for the BWO was made. A $9 \mathrm{~mm}$ hyperhemispherical lens was attached to the end of the test fixture. A cosine transition as described above, equation ( 3 ), was used to mate the lens to the slot line. Frequency response was studied both with and without a HDP focusing lens. The results are shown in figure 9 and are as good as the better Vivaldi horns studied in this paper, both in flatness and power response. Additionally, they do not display the absorption bands that plague the vivaldi family.

Polarization. - Finally, we have studied the polarization of the radiation from both the Vivaldi horns and the lens coupling system. In this study, polarizing filters were placed between the detector and the test fixture. Figure 10 shows a typical result. The polarization of the radiation transmitted by the lens is displayed. The radiation is strongly polarized in the plane of the horn and the slot line.

Tests at 85 to $100 \mathrm{GHz}$

Reference experiment. - Measurements in the 85 to $100 \mathrm{GHz}$ range were made using the setup shown in figure 3 


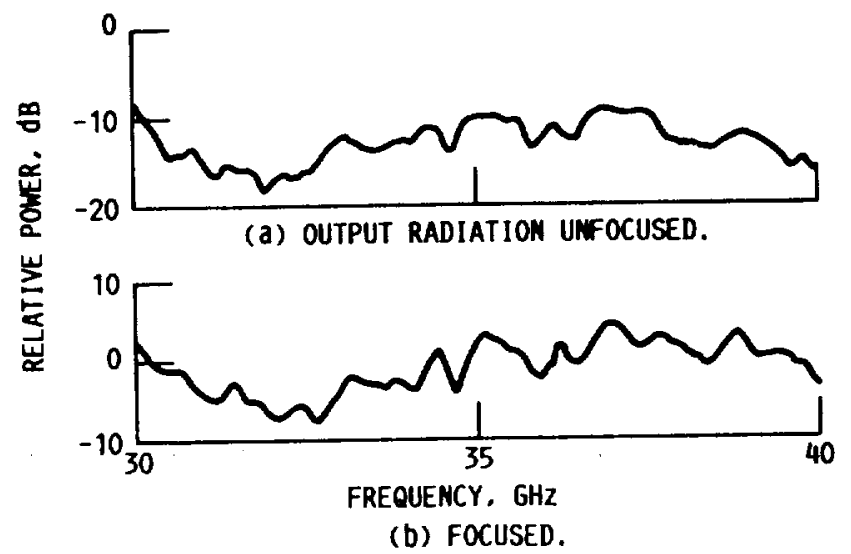

FIG. 9 POWER OUTPUT OF A SLOT LINE WITH A COSINE TRAMSITION FEEDING A HYPERHEMISPHERICAL LENS.

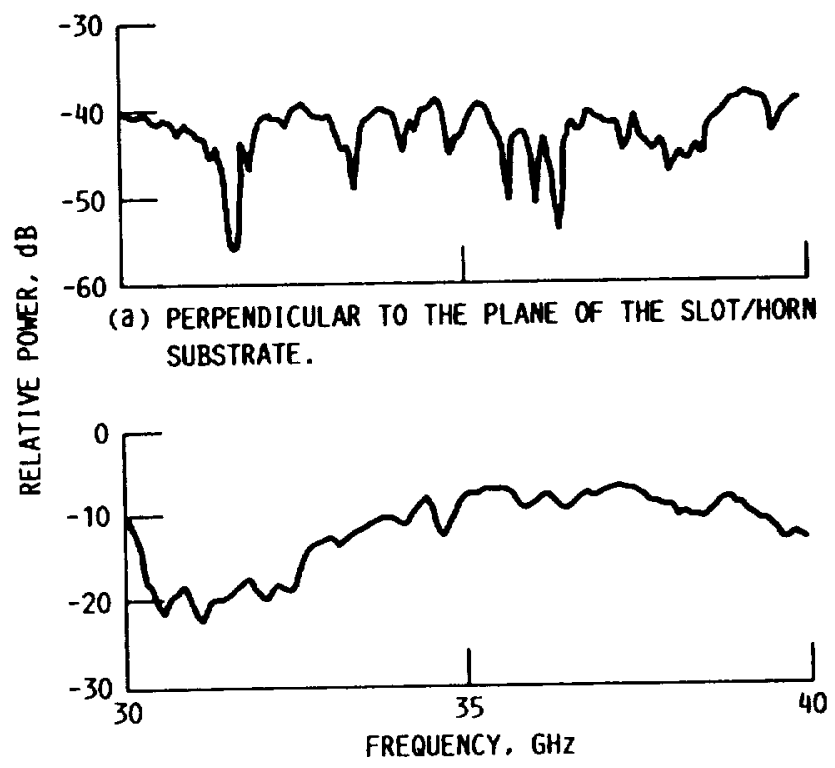

(b) Parallel to the Plane of the substrate.

FIG. 10 POLARIZATION OF RADIATION FROM TYPICAL VIVALDI HORN/LENS SYSTEM. 
and referenced to the curve in figure $4(c)$. A focusing lens was necessary because the detector was not sensitive to the lower signal power available at these higher frequencies. However, our results at 30 to $40 \mathrm{GHz}$ show that a focusing lens can be used without introducing standing wave frequency bands and without otherwise distorting the frequency response of the horn.

Comparison of planar horns. - The better Vivaldi horns identified at 30 to $40 \mathrm{GHz}$ were chosen for scaling to 85 to $100 \mathrm{GHz}$. These horns are 3 and $4 \lambda$ long (at $90 \mathrm{GHz}$ ) with opening's of $\lambda / 2$ and $\lambda$. Figure 11 compares the typical frequency response for the $4 \lambda$ horn. The average relative power measured is roughly the same for both horns although slightly higher for the $\lambda$ horn which also gives a flatter frequency response. The frequency response of both horns is disappointing and could not be substantially changed with dielectric covers. Figure $11(c)$ shows a typical trace with a dielectric on the horn with a $1 \lambda$ opening. Comparison with the bare horns in the same figure show little change in the frequency response. It is

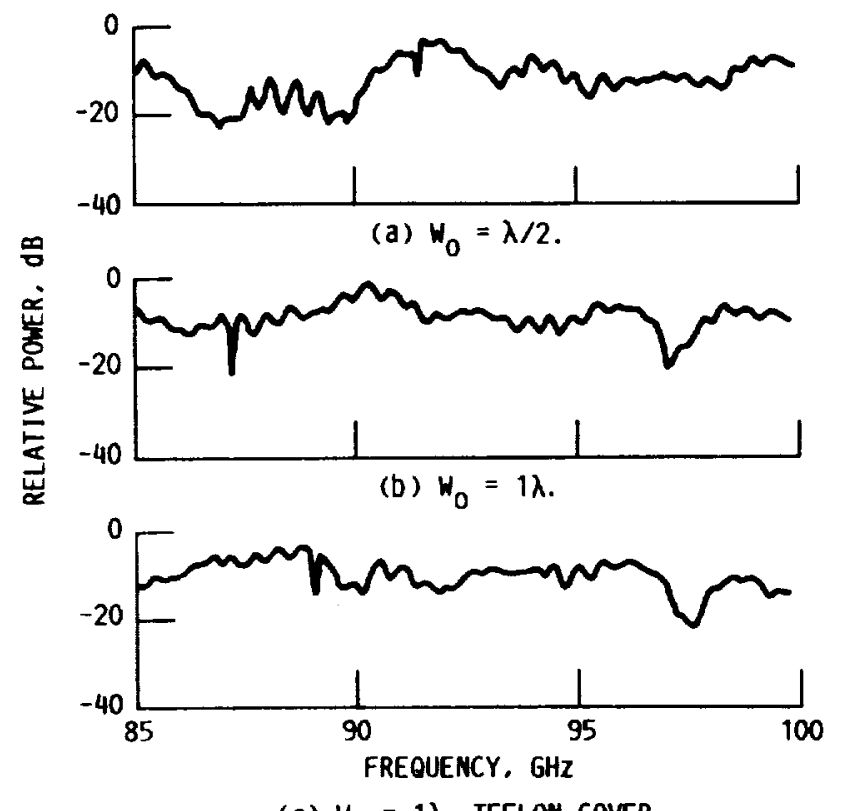

(c) $\omega_{0}=1 \lambda$; TEFLON COVER.

FIG. 11 RESPONSE OF VIVALDI HORN $(L=4 \lambda)$ WITH AND WITHOUT TEFLON COVER AT 85-100 GHz. 
interesting, however, to note that with the focusing lens removed and the detector situated very close to the horns, the dielectric again shows the same properties as those seen at 30 to $40 \mathrm{GHz}$. This is explained by noting that the electric field vector prefers a higher dielectric path and therefore is confined by the dielectric. Hence radiation that normally would travel above the horn is bent more toward the endfire direction and the detector. This is geometrically important at 30 to $40 \mathrm{GHz}$, but for our experiments at 85 to $100 \mathrm{GHz}$ the lens had to be near the horn and, therefore, it had already collected the radiation emitted above the horn. Metallic reflectors demonstrated that this is indeed the case.

Hyperhemispherical lens system. - Our data has indicated that Vivaldi horns have broad band frequency characteristics that are unsuitable for the intended BWO application. Our other candidate, the hyperhemispherical lens fed with a cosine transition fares much better at 85 to $100 \mathrm{GHz}$. We have made two types of measurements using the lens at these frequencies; first, the lens was mounted flush against the slot line with no mating transition, and second, with a cosine transition as discussed. The results are given in figure $12(a)$ and (b). It is seen that a mating transition is necessary between the slot line and the lens. The hyperhemispherical coupling lens is superior to the Vivaldi horns in both flatness and power response.

Linear tapered slot line. - As a final measurement a simple linear "V" transition, with an opening of $1 / 2 \lambda$ at a length of $3 \lambda$, was tested. The results are shown in figure $12(c)$. The literature suggests that this type horn should be little different from a vivaldi horn with similar dimensions. However, we found that the " $V$ " is far superior to the Vivaldi horns in both power response and flatness and appears to be a very good candidate for scaling to higher frequencies. The results suggest that the " $V$ " transition be incorporated with optical devices such as focusing lens to achieve maximum power at normal incidence over large frequency bands.

\section{High Frequency Experiment}

A preliminary study of the hyperhemispherical lens and collimating system shown in figure 2 was made at $337 \mathrm{GHz}$. This frequency is near the expected center frequency of the next experimental BWO which is soon to be constructed. 


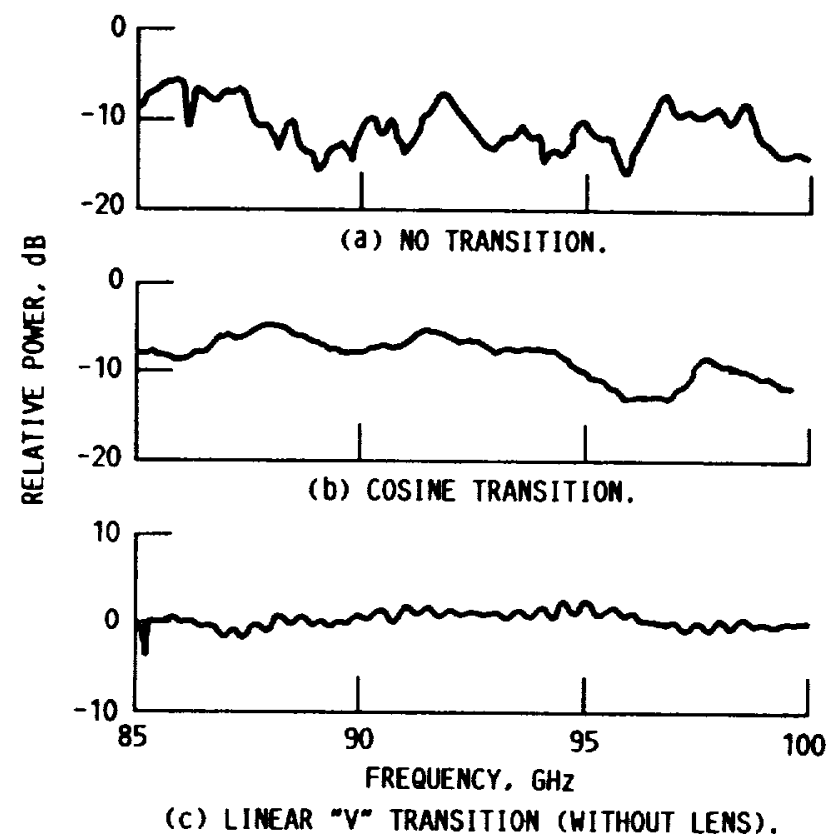

FIG. 12 HYPER-HEMISPHERICAL LENS FED BY SLOT LIME.

The collimating lens is a sapphire plano-convex lens with a $10 \mathrm{~mm}$ aperture and a $20 \mathrm{~mm}$ focal length (at $546.1 \mathrm{~nm}$ ). The index of refraction at $300 \mathrm{GHz}$ was taken from reference 9 to be equal to 3.064 .

The hyperhemispherical lens was ground from a $3 \mathrm{~mm}$ sapphire ball bearing. The lens behaves as an extremely fast lens if it is ground so that the ratio of the radius of the sphere to the shortened radius is equal to the index of refraction.

$$
f \text {-number }=\frac{n}{2\left(n^{2}-1\right)}
$$

For a refractive index of $n=3.064$ the $f$-number of the sapphire lens is 0.1826 . In order to simplify the grinding procedure the ratio of $R / d$ was taken to be 3 rather than 3.064. A ray trace of the lens system (fig. 2) indicates that the approximation is acceptable. 
A $\mathrm{CO}_{2}$ pumped FIR laser (Apollo 122) was used as the $337 \mathrm{GHz}$ source. The FIR resonator gas was $(1,1)$ Difluoroethylene and the $\mathrm{CO}_{2}$ laser was tuned to the 1OP(22) line. The emergent FIR line is linearly polarized in a plane which is $30^{\circ}$ from the horizontal.

A pyroelectric detector placed in the FIR laser beam was used to make a power measurement with and without the lens system. Only a $2 \mathrm{~dB}$ increase in detected signal was found when the lens system was used to focus the collimated laser beam. However, the FIR beam is fairly narrow and even without a focusing lens most of the energy is intercepted by the detector.

\section{Concluding Remarks}

The purpose of this study is to identify suitable output couplers for submillimeter wavelength backward-wave oscillators having large bandwidths. Because radiative couplers are desirable at these wavelengths, several schemes for making transitions between microstrip slot lines and free space were investigated. The application requires maximum power transfer and flat frequency response over a large bandwidth.

We found Vivaldi horns to have unsatisfactory resonances perhaps because the horns we studied were "short" horns having lengths of 3 and $4 \lambda$ rather than the usual $10 \lambda$. The limitation on horn length arose from the limited space available on the substrate of the etched slow-wave circuit.

However we have found several techniques, to maximize and to "flatten" the frequency response of these horns. A dielectric cover was used to confine the radiation and to direct it toward the endfire direction. Thick dielectrics will also eliminate some of the resonance bands. We have found that the smaller openings for the horns are much better than the larger horn openings.

By incorporating optical techniques, i.e., focusing lenses, signal strengths can be improved without sacrificing the frequency response of the horns.

We found alternate coupling schemes to be superior to Vivaldi horns. In particular: a simple linear " $V$ " antenna has a frequency/power characteristic which is superior to a 
Vivaldi of similar dimensions; a hyperhemispherical lens fed with a cosine transition between the slot line and the lens is also an effective method to couple energy out of the BWO.

The hyperhemispherical gathering lens and collimator system was studied at $337 \mathrm{GHz}$ near the expected frequency of the next experimental BWO which is presently under construction. A $2 \mathrm{~dB}$ increase of signal was found. However, the FIR laser beam is somewhat narrow and even without the lens system most of the energy is intercepted by the detector.

\section{REFERENCES}

1. L. Teyssier and B. Epsztein, "Advances in Submillimeter Carcinotrons," Eighth International Conference on Infrared and Millimeter Waves, IEEE, Piscataway, NJ, 1983, paper W2.2.

2. M.B. Golant, Z.T. Alekseenko, 2.S. Korotkova, L.A. Lunkina, A.A. Negirev, O.P. Petrova, T.B. Rebrova, V.S. Savel'ev, "Wide-Range Oscillators for the Submillimeter Wavelengths," Instrum. Exp. Tech. (Engl. Trans1.), No. 3, May 1969, pp. 801-802 (1969).

3. N.N. Efremow, M.W. Geis, D.C. Flanders, G.A. Lincoln, and N.P. Economou, "Ion-Beam-Assisted Etching of Diamond," J. Vac. Sci. Technol. B., vol. 3, no. 1, Jan.-Feb. 1985, pp. 416-418.

4. L.R. Barnett, J.M. Baird, R.W. Grow, and S.G. Holmes, "Submillimeter-Wave BWO's," 1985 International Electronic Devices Meeting (IEDM) Technical Digest, IEEE, Piscataway, NJ, 1985, pp. 364-365.

5. P.J. Gibson, "The Vivaldi Aerial," Conference Proceedings: 9th European Microwave Conference, Microwave Exhibitions and Publishers Ltd., Kent, England, 1979, pp. 101-105.

6. R. Janaswamy and D.H. Schaubert, "Analys is of the Tapered Slot Antenna," IEEE Trans. Antennas Propagat., vol. 35, no. 9, Sept. 1987, pp. 1058-1065. 
7. R. Simons, NASA Lewis Research Center, Cleveland, $\mathrm{OH}$, private communication.

8. S.B. Cohn, "Slot Line on a Dielectric Substrate," IEEE Trans. Microwave Theory Techniques, vol. 17, no. 10, oct. 1969, pp. 768-778.

9. M.N. Afsar and K.J. Button, "Millimeter-Wave Dielectric Properties of Materials," Infrared and Millimeter Waves, Vol. 12, Electromagnetic Waves in Matter, Part II, K.J. Button, ed., Academic Press, New York, 1984, pp. $1-42$. 


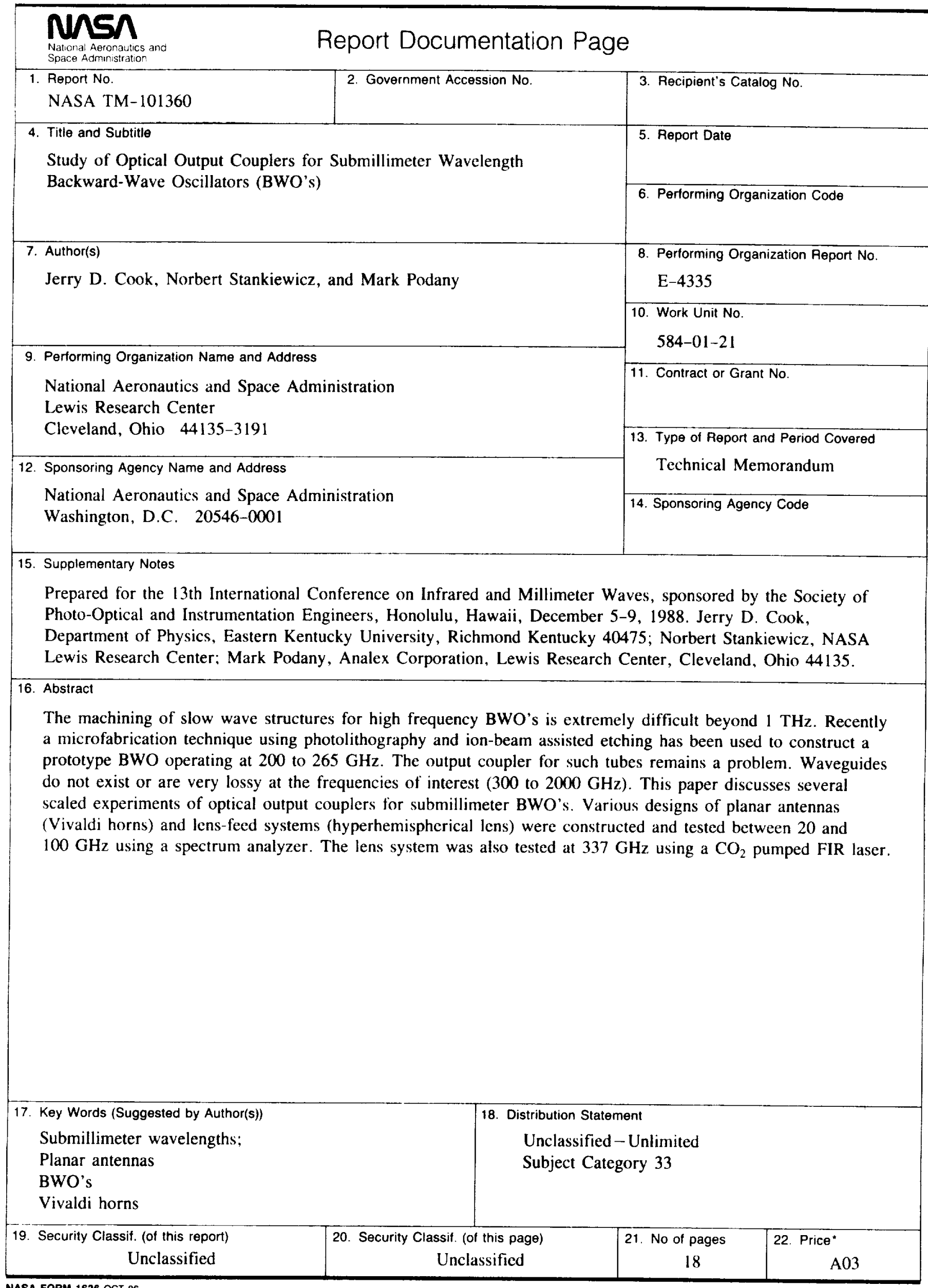


National Aeronautics and

Space Administration

Lewis Research Center

Cleveland. Ohio 44135

\section{Orialed Buinew}

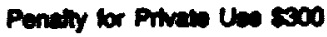

SECOND CLASS MAIL

\section{ADDRESS CORRECTION REQUESTED}

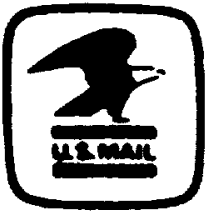

Poetage and Fees Paid National Aeronautics and

Space Administration NASA-451 